\title{
OPEN
}

\section{Longitudinal change in everyday function and behavioral symptoms in frontotemporal dementia}

Claire M. O'Connor, MOT (Hons); Lindy Clemson, PhD; Michael Hornberger, PhD; Cristian E. Leyton, PhD; John R. Hodges, MBBS, MD; Olivier Piguet, PhD; Eneida Mioshi, PhD

\section{Abstract}

Background: The relationship between behavioral changes and functional decline in frontotemporal dementia (FTD) is not well understood. Methods: Thirtynine patients ( 21 behavioral variant FTD [bvFTD], 18 semantic variant primary progressive aphasia [svPPA]) were followed up longitudinally (2-4 years follow-up). Functional (Disability Assessment for Dementia) and behavioral (Cambridge Behavioural Inventory Revised) assessments were included for between-group (pairwise comparisons, mixed model analysis) and within-group analyses (bivariate correlations). Results: Functionally, patients with bvFTD were more impaired than patients with svPPA at baseline and continued to be at follow-up, despite similar disease duration. By contrast, behavioral impairments differed between patient groups at baseline and at follow-up. At baseline, patients with

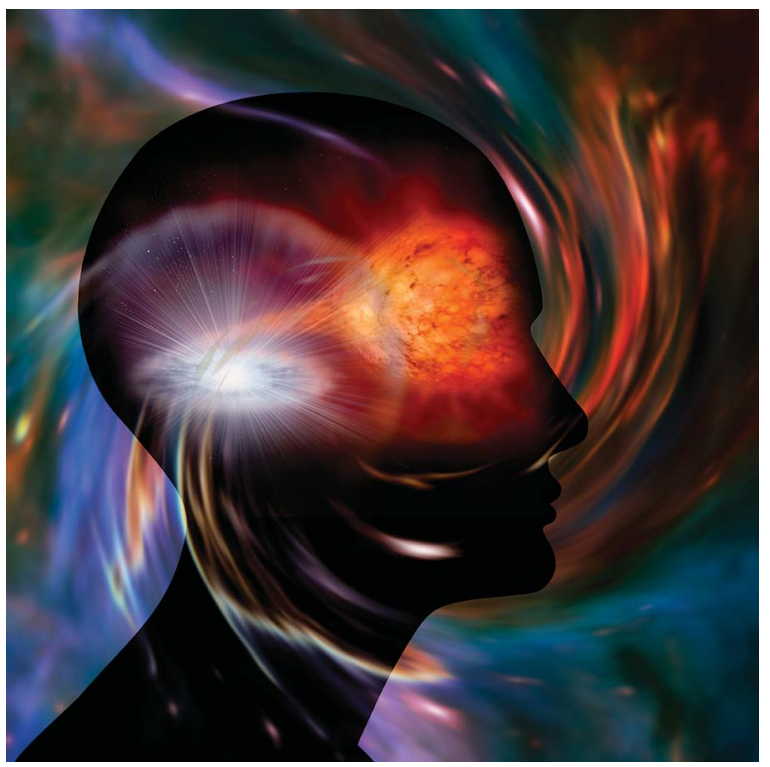
bvFTD exhibited higher levels of apathy and changes in eating than patients with SvPPA; disinhibited and stereotypical behaviors were similar. Over the years, patients with bvFTD showed reduction in disinhibition and stereotypical behavior while apathy and eating changes increased. By contrast, all measured behaviors increased in patients with svPPA over time. Finally, only apathy made longitudinal contributions to functional disability in patients with svPPA, whereas apathy and stereotypical behavior were associated with increased disability in patients with bvFTD. Conclusions: Despite shared overlapping baseline behavioral symptoms, patients with bvFTD are more functionally impaired than patients with svPPA. Apathy has a strong role in disability for both bVFTD and svPPA, but stereotypical behaviors only contributed to functional deficits in

Ageing, Work \& Health Research Unit (CMO, LC) and Speech Pathology (CEL), Faculty of Health Sciences, University of Sydney, Australia; Faculty of Medicine and Health Sciences (MH, EM), University of East Anglia, UK; Neuroscience Research Australia (CEL, JRH, OP); and ARC Centre of Excellence in Cognition and its Disorders (JRH, OP) and School of Medical Sciences (JRH, OP), University of New South Wales, Australia. Funding information and disclosures are provided at the end of the article. Full disclosure form information provided by the authors is available with the full text of this article at Neurology.org/cp. The Article Processing Charge was paid by the Alzheimer Association.

This is an open access article distributed under the terms of the Creative Commons Attribution-NonCommercialNoDerivatives License 4.0 (CC BY-NC-ND), which permits downloading and sharing the work provided it is properly cited. The work cannot be changed in any way or used commercially.

Correspondence to: e.mioshi@uea.ac.uk 
patients with bvFTD. Our findings suggest that rigid/compulsive behaviors may in fact support activity engagement in patients with svPPA. Taken together, our results indicate that interventions to reduce disability in the FTD spectrum require an alternative rationale in comparison to Alzheimer disease dementia, and should carefully weigh the interaction of behavioral symptoms and functional status. Neurol Clin Pract 2016;6:419-428

$\mathrm{F}$

rontotemporal dementia (FTD) impinges markedly on everyday function ${ }^{1-3}$ but the degree and nature of functional disability depends on the clinical presentation. Specifically, a gradient of functional disability exists across the 3 primary clinical FTD subtypes: patients with behavioral variant FTD (bvFTD) are severely impaired, followed by nonfluent variant primary progressive aphasia (nfvPPA) and semantic variant primary progressive aphasia (svPPA). ${ }^{1,4}$ Despite functional differences between bvFTD and svPPA, patients can share similar behavioral profiles; yet for patients with svPPA, this is often less noted due to the severe and prevalent language and semantic deficits. ${ }^{5,6} \mathrm{nfvPPA}$, on the other hand, elicits a comparatively mild behavioral profile, and for this reason it was not included in the present study.

The nature of functional disability in FTD variants is likely to be a direct reflection of the specific behavioral and cognitive changes seen in each syndrome. ${ }^{2,7}$ There is well-reported overlap of behavioral symptoms between bvFTD and svPPA., ${ }^{5,6}$ It is critical to investigate the role of behavioral changes in functional disability; once independent factors are identified, novel interventions can be developed to reduce disability.

We hypothesized that similar levels of behavioral symptoms across bvFTD and svPPA would be present, but their specific contribution to functional disability would differ. The study aimed to (1) determine baseline function and behavior status in bvFTD and svPPA; (2) examine longitudinal functional and behavioral changes; and (3) examine relationships between functional and behavioral changes longitudinally.

\section{METHODS}

\section{Participants}

Thirty-nine individuals diagnosed with FTD $($ bvFTD $=21$; svPPA $=18)$ and their caregivers were recruited from FRONTIER, the frontotemporal dementia research group based in Sydney, Australia. All patients met current criteria for either possible or probable bvFTD or $\mathrm{svPPA}^{8,9}$ and diagnoses were based on a multidisciplinary consensus (neurologist and neuropsychologist). Study participants were assessed in the clinic, home, or care facility on 2-4 separate occasions approximately 16 months between each assessment (mean $16.4 \pm 8.2$ ) between November 2007 and January 2012, with 200 individual assessments completed in total.

Participants were included if they had a close relative or friend informant who could reliably report on the person's behavior and everyday routine, did not have major depression, and did not have any physical disability that could influence ability to undertake activities of daily living (ADLs). The ADL measure included in this study was not used in the diagnostic process. Disease duration was estimated from the onset of symptoms as described by the informant at the time of diagnosis.

Standard protocol approvals, registrations, and patient consents This study was approved by the University of New South Wales and the South Eastern Sydney and Illawarra Area Health Service human ethics committees. Written informed consent was obtained from each patient or primary caregiver where appropriate.

\section{Instruments}

Functional assessment ADLs were assessed with the Disability Assessment for Dementia (DAD),${ }^{10}$ an informant-based scale made up of 17 items pertaining to basic ADLs and 23 to instrumental ADLs. Basic ADL items include hygiene, dressing, continence, and eating; 
instrumental $\mathrm{ADL}$ items include meal preparation, telephoning, going on an outing, finance and correspondence, medications, and leisure and housework. In order to avoid bias towards certain activities (e.g., meal preparation or housework), nonapplicable questions are omitted from the final score and the score is reported as a percentage. Greater impairment is indicated by lower DAD scores.

In order to further distinguish baseline clinical differences between the groups, basic ADL and instrumental ADL subscores were included in the analysis in addition to total DAD scores. All caregivers were interviewed by a research occupational therapist (EM or $\mathrm{CO}$ ) at baseline and at each follow-up visit.

Behavioral assessment The Cambridge Behavioural Inventory Revised (CBI-R $)^{11}$ is a caregiver-completed assessment that comprises 45 items assessing behavior across 10 domains. Each question requires the caregiver to rate behavior on a 5-point scale $(0=$ never, 1 = a few times per month, 2 = a few times per week, $3=$ daily, and $4=$ constantly), with higher scores indicating higher frequency of abnormal behavior. Data for 4 behavioral subsections commonly associated with FTD ${ }^{9,12,13}$ were used: disinhibition (e.g., exhibiting socially embarrassing behavior; making inappropriate comments), stereotypical behaviors (e.g., adhering to rigid daily routines; being obsessive about watching the time), apathy (e.g., lacking motivation or enthusiasm; appearing indifferent), and eating changes (e.g., eating more sweet/fatty foods then before; declining table manners such as stuffing food into the mouth; eating an extremely restricted diet of specific foods). Subsection scores were converted to percentages to allow for domain comparison; higher scores represent greater behavioral change.

General cognitive assessment At baseline, all participants were administered the Addenbrooke's Cognitive Examination Revised (ACE-R). ${ }^{14}$ The ACE is a brief screening tool sensitive to the early stages of dementia, which assesses 5 cognitive domains: attention and orientation, memory, verbal fluency, language, and visuospatial abilities. The total score is 100 , where lower scores indicate more impaired cognitive functioning. The cutoff of 88/100 yields $89 \%$ specificity and $94 \%$ sensitivity for diagnosing dementia.

\section{Data analyses}

Statistical analyses were performed using SPSS 21.0 (IBM, Armonk, NY) for Windows. Demographic data across both patient groups were compared using parametric independent sample $t$ tests and $\chi^{2}$ tests for sex comparisons. Normality of distribution of baseline variables was assessed using Kolmogorov-Smirnov tests, and due to skewed data, nonparametric measures (Mann-Whitney $U$ ) were used for pairwise comparisons at baseline. To examine changes in function and behavior between the diagnostic categories over time, linear mixed-effect models were applied. Mixed model analyses allow for accounting for missing data, ${ }^{15}$ which was relevant in the current data, as the number of people who had follow-up assessments declined with each year. DAD data for bvFTD declined across the 4 visits as follows: $\mathrm{n}=21, \mathrm{n}=16, \mathrm{n}=6$, and $\mathrm{n}=2$; for svPPA: $\mathrm{n}=18, \mathrm{n}=10, \mathrm{n}=10$, and $\mathrm{n}=4$. CBI-R data for bvFTD declined across visits: $\mathrm{n}=21, \mathrm{n}=20, \mathrm{n}=8$, and $\mathrm{n}=5$; for svPPA: $\mathrm{n}=18, \mathrm{n}=15, \mathrm{n}=11$, and $\mathrm{n}=5$. Fixed effects in the model included time, diagnostic category (bvFTD or svPPA), and the interaction between time and diagnostic category. Individual patient variability at baseline was the only random effect included; hence a random intercept was included in each model. Both the fixed and random effects in the model determined the variability of any estimated parameters. Separate models were built for each of the following dependent variables: DAD total, DAD instrumental ADLs, DAD basic ADLs, CBI-R Disinhibition, CBI-R Stereotypical behavior, CBI-R Apathy, and CBI-R Eating changes. Due to the small sample size, a linear first-order polynomial was applied in the analysis. For any given model, a significant effect for time would indicate that the dependent variable changes over time, and a significant interaction between time and diagnostic category would indicate that bvFTD and svPPA have different 
Table 1 Demographic characteristics and behavioral and functional outcomes in behavioral variant frontotemporal dementia (bvFTD) and semantic variant primary progressive aphasia (svPPA)

\begin{tabular}{|c|c|c|c|c|}
\hline & bvFTD (n = 21) & svPPA (n = 18) & Statistic test & p Values \\
\hline Age, y & $62.1(10.5)$ & $63.7(7.8)$ & $t=-1.095$ & $N S^{a}$ \\
\hline Sex, M/F & $14 / 7$ & $12 / 6$ & $x^{2}=0.116$ & $N S^{b}$ \\
\hline Disease duration, y & $3.5(2.4)$ & $4.6(2.3)$ & $t=-1.512$ & $N S^{a}$ \\
\hline Baseline ACE-R score, $\max 100$, cutoff $88 / 100$ & $74.7(10.0)$ & $56.8(15.6)$ & $t=4.173$ & $<0.001^{a}$ \\
\hline DAD: Instrumental ADLs & $35(9-46)$ & $81.8(45-90)$ & $U=40$ & $<0.005^{c}$ \\
\hline DAD: Basic ADLs & $64.7(57-88)$ & $94.1(76-100)$ & $U=53$ & $<0.01^{\mathrm{c}}$ \\
\hline CBI-R: Disinhibition & $33.3(19-60)$ & $16.7(3-39)$ & $U=121.5$ & $N S^{c}$ \\
\hline CBI-R: Stereotypical behavior & $56.3(34-78)$ & $46.9(5-81)$ & $U=163$ & $\mathrm{NS}^{\mathrm{c}}$ \\
\hline $\begin{array}{l}\text { Abbreviations: ACE-R = Addenbrooke's Cognitive Ex } \\
\text { Disability Assessment for Dementia; NS = not sign } \\
\text { Disease duration refers to the time between sympt } \\
\text { a Independent samples } t \text { test. Scores are means (SD }\end{array}$ & \multicolumn{4}{|c|}{$\begin{array}{l}\text { Disease duration refers to the time between symptom onset and baseline assessment date. } \\
\text { a Independent samples } t \text { test. Scores are means (SD). }\end{array}$} \\
\hline${ }^{\mathrm{b}} \chi^{2}$ test using Yates correction. & 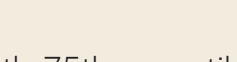 & - & & \\
\hline
\end{tabular}

changes in the dependent variable over time. To examine longitudinal relationships between behavioral variables and ADL function within groups, subject means for each $\mathrm{CBI}$ and DAD variable were calculated across observations. Pearson correlation was then calculated between these subject means and a weighted correlation coefficient was applied to account for the different number of observations across subjects. ${ }^{16}$ Bonferroni corrections were applied for multiple comparisons.

\section{RESULTS}

\section{Demographics}

The 2 clinical groups were matched for age, sex distribution, education, and disease duration. The bvFTD group scored higher than the svPPA group on global cognition ACE-R, reflecting the language load of this task (table 1).

\section{Baseline assessment}

Pairwise comparisons revealed that patients with bvFTD scored lower than the svPPA group at baseline across all ADL scores (table 1) despite matched disease duration.

To investigate degrees of behavioral changes between groups, CBI-R percentage scores were categorized as absent (score $=0) ;$ mild/moderate $($ score $=1-50)$; or severe/very severe (score $=$ 51-100). As shown in figure $1, \mathrm{~A}$ and $\mathrm{B}$, levels of disinhibition and stereotypical behavior were similar for bvFTD and svPPA. In contrast, patients with bvFTD had higher scores for apathy and eating changes, reflecting more marked neuropsychiatric changes in this subgroup (table 1). A striking group difference was detected in regards to apathy (table 1), where $71.5 \%$ of patients with bvFTD exhibited severe/very severe apathy compared with $33.3 \%$ of patients with svPPA (figure 1C). Changes in eating behavior were present in $100 \%$ of patients with bvFTD, with half of them being reported as presenting with severe/very severe changes, 
Figure 1 Percentage distribution according to severity of baseline behavioral changes in behavioral variant frontotemporal dementia (bvFTD) and semantic variant primary progressive aphasia (svPPA) cohorts

A Disinhibition

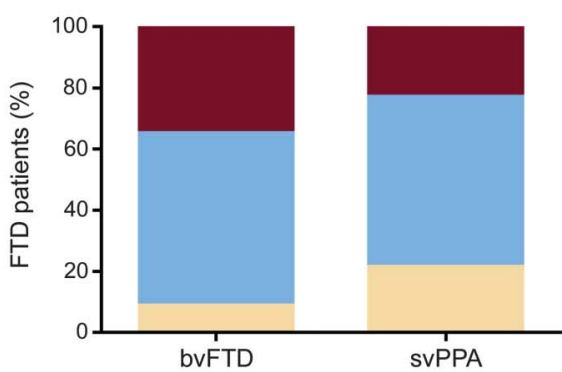

C Apathy

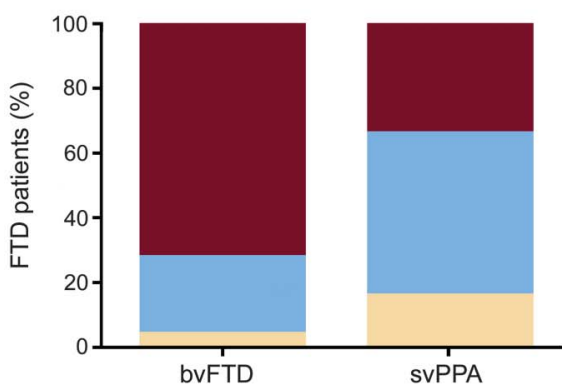

B

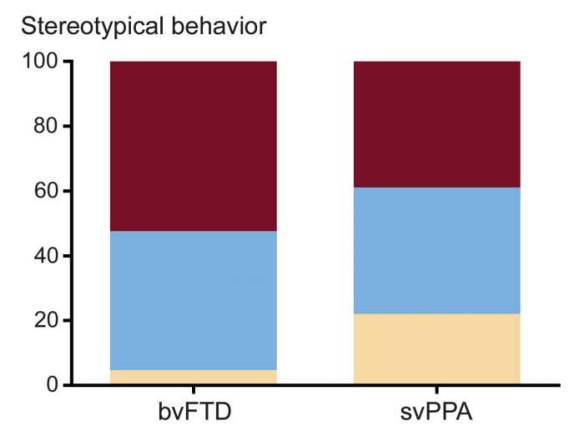

Absent

Mild/

Moderate

Severe/ Very severe

(A-D) Behavioral change absent, mild/moderate, or severe/very severe.

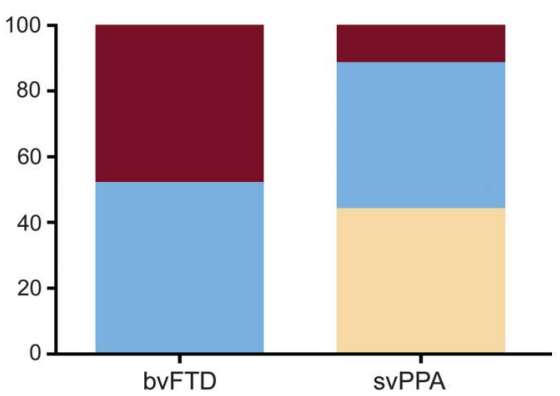

whereas alterations in eating habits were reported in only $56 \%$ of patients with svPPA and the majority were mild/moderate changes (figure 1D).

\section{Longitudinal changes}

ADL function declined over time in both groups, with bvFTD worse than svPPA on all DAD scores: total ADLs $\left(F_{1,53.09}=79.30, p<0.001\right)$; instrumental ADLs $\left(F_{1,54.91}=48.78, p<\right.$ $0.001)$; and basic ADLs $\left(F_{1,52.92}=54.87, p<0.001\right)$. For basic ADLs specifically, patients with bvFTD exhibited a more rapid decline than patients with $\operatorname{svPPA}\left(F_{1,52.92}=4.57, p<\right.$ 0.05 ; figure $2 \mathrm{C}$ ). Of note, the bvFTD group was near floor performance on the total DAD by year 3 (figure 2A), while patients with svPPA were still performing at around 55\% of ability in relation to their premorbid levels.

Longitudinal changes in levels of disinhibition $\left(F_{1,72.84}=11.05, p<0.005\right)$ and stereotypical behavior $\left(F_{1,72.46}=11.28, p<0.005\right)$ differed between bvFTD and svPPA despite the 2 groups showing similar levels on these measures at baseline. svPPA demonstrated increasing levels of these behaviors over time, whereas both behaviors declined in bvFTD (figure $2, \mathrm{D}$ and $\mathrm{E})$. Levels of apathy $\left(F_{1,75.34}=16.61, p<0.001\right)$ and eating changes $\left(F_{1,74.68}=26.79, p<0.001\right)$ increased at a similar rate over time for both groups; however, patients with bvFTD continued to exhibit higher levels of these behaviors than patients with svPPA (figure 2, F and G).

\section{Longitudinal correlations}

Pearson correlations using subject means and weighted correlation coefficients were conducted to examine relationships between decline in ADL function (DAD subscores) and behavioral changes (CBI behavioral scores) over time (table 2). For bvFTD, decline in both total ADL and instrumental ADL scores were associated with apathy $(r=-0.512, p<0.001 ; r=$ $-0.586, p<0.001$ ), while decline in basic ADLs was correlated with stereotypical behavior 


\section{Marked overlapping behavioral changes in} bvFTD and svPPA dissociate over time:

\section{behavioral changes become less pronounced in} bvFTD but increase in SVPPA.

$(r=0.329, p<0.015)$. For svPPA, apathy correlated with both total ADLs $(r=-0.514$, $p<0.005)$ and instrumental ADLs $(r=-0.583, p<0.001)$.

\section{DISCUSSION}

Marked overlapping behavioral changes in bvFTD and svPPA dissociate over time: behavioral changes become less pronounced in bvFTD but increase in svPPA. Importantly, decline in everyday function was associated with increased apathy in bvFTD and svPPA. Stereotypical behavior and $\mathrm{ADL}$ decline was only associated with disability in patients with bvFTD, despite comparable levels of stereotypical behavior at baseline in both groups. In comparison, disinhibition per se did not seem to make a direct contribution to functional decline over time for either group. Taken together, our results confirm previous findings of more severe functional impairment in bvFTD than in svPPA, and we confirmed that this difference in functional status continues as the disease progresses.

The correlation between apathy and worsening function demonstrated in both bvFTD and svPPA is perhaps not surprising given that as apathy increases, a person is less likely to

Figure 2 Model representations of performance on functional and behavioral measures over time for behavioral variant frontotemporal dementia (bvFTD) and sematic variant primary progressive aphasia (svPPA) (plots model representations of scores)

B. Instrumental $A D L$ function

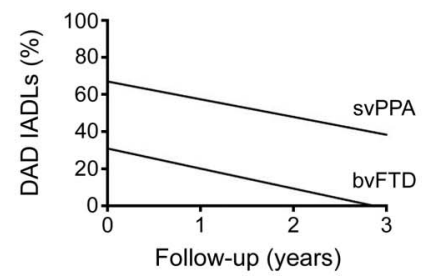

E. Stereotypical behavior

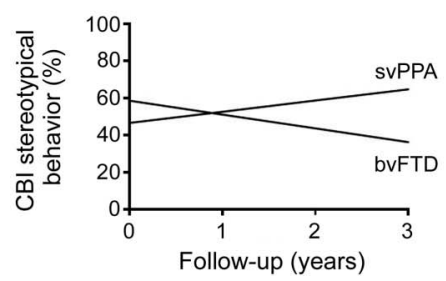

A. Total ADL function

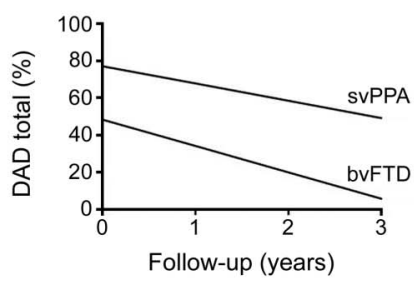

C. Basic ADL function

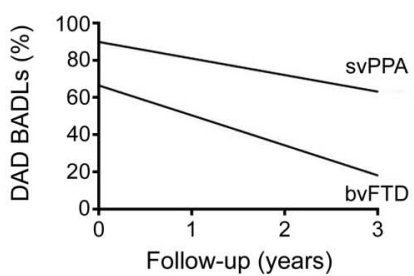

F. Apathy

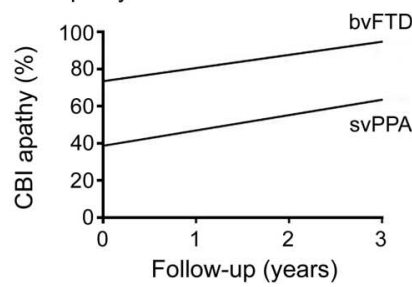

D. Disinhibition

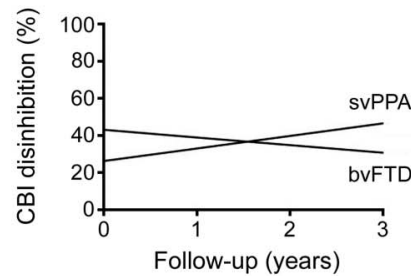

G. Eating changes

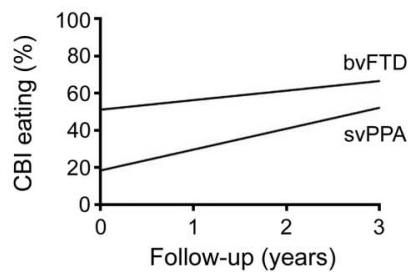

(A-G) $A D L=$ activities of daily living; $B A D L=$ basic activities of daily living; $C B I=$ Cambridge Behavioural Inventory; $\mathrm{DAD}=$ Disability Assessment for Dementia; IADL = instrumental activities of daily living. 


\section{Across both patient groups, disinhibition did not play a major role in functional impairment.}

participate in daily activities. This association has also been reported in Alzheimer disease (AD), where apathy, anxiety, and aberrant motor disturbance correlate with functional disability. ${ }^{17-19}$ The longitudinal link between apathy and functional decline in bvFTD reported here also supports findings from previous cross-sectional studies. ${ }^{2,7}$

Across both patient groups, disinhibition did not play a major role in functional impairment. By contrast, apathy, sometimes regarded as the flip side of disinhibition, was detrimental to functional activities as patients failed to initiate them. In the present study, apathy and changes in eating habits continue to increase throughout progression in both bvFTD and svPPA. In contrast, disinhibited and stereotypical behaviors, which are often difficult to manage, ameliorate over time in bvFTD while continuing to worsen in svPPA. A possible reason for this may be the differing rates of disease progression: patients with bvFTD decline faster into frank apathy, which may restrict the concurrent expression of other symptoms, while patients with svPPA have a protracted disease progression. Past studies have reported a pattern of behavioral fluctuation over time in bvFTD, where patients may became more docile and compliant as some challenging behaviors disappear throughout disease progression, ${ }^{20}$ or new behaviors appear in later stages. ${ }^{6,21-23}$ Comparable findings of behavioral fluctuations along dementia progression have been described in the $\mathrm{AD}$ dementia literature, ${ }^{24,25}$ reflecting the complex nature of neuropsychiatric symptoms in neurodegenerative diseases.

The contribution of stereotypical behaviors to function seems to vary in bvFTD and svPPA. In bvFTD, decline in stereotypical behaviors was associated with a reduction of participation in basic daily functions. This association was not detected in svPPA, possibly due to the noteworthy increase of these behaviors over time. In fact, our results suggest that the presence of stereotypical behaviors may serve as a compensatory support for continued participation in rigid self-care routine tasks in patients with svPPA, which in turn show very mild decline even after 4 years. ${ }^{26,27}$ Closer examination at the specific dysfunctional behaviors reveals that the nature of stereotypical behaviors is different. Patients with bvFTD are more likely to exhibit impulsive behaviors, such as clapping or counting aloud, whereas patients with svPPA are prone to adhere to a rigid routine and clock-watching. ${ }^{28,29}$ Similarly, longitudinal changes in eating habits in svPPA seem to mirror their stereotypical behavior: rigid routines and bizarre choices

Table 2 Correlation matrix for repeated measures using subject means

\begin{tabular}{|c|c|c|c|c|c|c|}
\hline \multirow[b]{2}{*}{ CBI-R variables } & \multicolumn{3}{|l|}{ bvFTD } & \multicolumn{3}{|l|}{ svPPA } \\
\hline & Total DAD & IADLs & BADLs & Total DAD & IADLs & BADLs \\
\hline Disinhibition, $r$ & -0.200 & -0.290 & -0.092 & -0.107 & -0.252 & 0.138 \\
\hline Stereotypical behavior, $r$ & 0.214 & 0.081 & $0.329^{a}$ & 0.079 & -0.091 & 0.342 \\
\hline Apathy, $r$ & $-0.512^{b}$ & $-0.586^{b}$ & -0.317 & $-0.514^{a}$ & $-0.583^{b}$ & -0.348 \\
\hline \multicolumn{7}{|c|}{$\begin{array}{l}\text { Abbreviations: } \mathrm{BADL}=\text { basic activities of daily living; bvFTD = behavioral variant frontotemporal } \\
\text { dementia; } \mathrm{CBI}-\mathrm{R}=\mathrm{Cambridge} \text { Behavioural Inventory-Revised; DAD = Disability Assessment for } \\
\text { Dementia; IADL = instrumental activities of daily living; svPPA = semantic variant primary pro- } \\
\text { gressive aphasia. }\end{array}$} \\
\hline
\end{tabular}


around food commonly develop within the first few years in svPPA. ${ }^{27,30-32}$ Overeating is a common early feature in bvFTD and more so than in svPPA, ${ }^{33}$ which may be more related to lack of inhibitory control. An interesting question for future research would be to confirm if the rigid/ compulsive nature of svPPA behaviors is more conducive to ADL performance, in particular for basic activities. Indeed, patients with svPPA remain relatively independent in everyday tasks for a much longer period of time, in line with a much more protracted disease progression. ${ }^{4,34,35}$

With disease progression, substantial overlap between FTD clinical variants arises, where merging clinical characteristics between bvFTD and svPPA have been well-described. ${ }^{23,36}$ Findings from this study highlight the early overlap of behavioral changes in both subgroups, which has a direct repercussion on family caregiver burden ${ }^{37}$ that increases noticeably in svPPA. ${ }^{38}$

This study has some limitations, including the relatively small numbers of participants, with declining numbers at each follow-up visit. Attrition of participants at follow-up is a common phenomenon as patients become too impaired to return to research centers, and the small numbers included in this study could limit the generalizability of the results. The primary reasons behind attrition were participants becoming too impaired to be followed up at the annual visits or participants' death. The use of the mixed model analysis provided more flexibility around this limitation in the data. The use of informant report measures could be a potential bias if caregivers underreport or overreport functional abilities and behaviors, ${ }^{39}$ but other studies have shown a great benefit of using them with bvFTD groups. ${ }^{40}$

Overlapping marked behavioral changes are very well known in bvFTD and perhaps currently less emphasized in svPPA. ${ }^{12}$ The present study offers novel insights into their distinct progression as well as diverse contribution to functional decline, where apathy is a clear negative contributor to disability to both bvFTD and svPPA. This finding alone has clinical implications, as apathy could be an important consideration in the development of interventions to improve functional performance in patients with FTD. Other factors underpinning disability in svPPA are yet to be further investigated, but our findings suggest that the development of treatments to slow functional decline in FTD (pharmacologic and nonpharmacologic) will need to consider the specific contributions of neuropsychiatric symptoms to disability.

\section{REFERENCES}

1. Mioshi E, Kipps CM, Dawson K, Mitchell J, Graham A, Hodges JR. Activities of daily living in frontotemporal dementia and Alzheimer disease. Neurology 2007;68:2077-2084.

2. Mioshi E, Kipps CM, Hodges JR. Activities of daily living in behavioural variant frontotemporal dementia. Alzheimer Dis Assoc Disord 2009;23:70-76.

3. Wicklund AH, Johnson N, Rademaker A, Weitner BB, Weintraub S. Profiles of decline in activities of daily living in non-Alzheimer dementia. Alzheimer Dis Assoc Disord 2007;21:8-13.

4. Mioshi E, Hsieh S, Savage S, Hornberger M, Hodges JR. Clinical staging and disease progression in frontotemporal dementia. Neurology 2010;74:1591-1597.

5. Banks SJ, Weintraub S. Neuropsychiatric symptoms in behavioural variant frontotemporal dementia and primary progressive aphasia. J Geriatr Psychiatry Neurol 2008;21:133-141.

6. Marczinski CA, Davidson W, Kertesz A. A longitudinal study of behavior in frontotemporal dementia and primary progressive aphasia. Cogn Behav Neurol 2004;17:185-190.

7. Kipps CM, Mioshi E, Hodges JR. Emotion, social functioning and activities of daily living in frontotemporal dementia. Neurocase 2009;15:182-189.

8. Gorno-Tempini ML, Hillis AE, Weintraub S, et al. Classification of primary progressive aphasia and its variants. Neurology 2011;76:1006-1014.

9. Rascovsky K, Hodges JR, Knopman D, et al. Sensitivity of revised diagnostic criteria for the behavioural variant of frontotemporal dementia. Brain 2011;134:2456-2477.

10. Gelinas I, Gauthier L, McIntyre M, Gauthier S. Development of a functional measure for persons with Alzheimer's disease: the disability assessment for dementia. Am J Occup Ther 1999;53:471-481.

11. Wear HJ, Wedderburn CJ, Mioshi E, et al. The Cambridge Behavioural Inventory-Revised. Demen Neuropsychologia 2008;2:102-107.

12. Bozeat S, Gregory CA, Ralph MAL, Hodges JR. Which neuropsychiatric and behavioural features distinguish frontal and temporal variants of frontotemporal dementia from Alzheimer's disease? J Neurol Neurosurg Psychiatry 2000;69:178-186. 
13. Liu W, Miller BL, Kramer JH, et al. Behavioral disorders in the frontal and temporal variants of frontotemporal dementia. Neurology 2004;62:742-748.

14. Mioshi E, Dawson K, Mitchell J, Arnold R, Hodges JR. The Addenbrooke's Cognitive Examination Revised (ACE-R): a brief cognitive test battery for dementia screening. Int J Geriatr Psychiatry 2006; 21:1078-1085.

15. Cnaan A, Laird NM, Slasor P. Tutorial in biostatistics: using the general linear mixed model to analyse unbalanced repeated measures and longitudinal data. Stat Med 1997;16:2349-2380.

16. Bland JM, Altman DG. Calculating correlation coefficients with repeated observations: part 2: correlation between subjects. BMJ 1995;310:633.

17. Boyle PA, Malloy PF, Salloway S, Cahn-Weiner DA, Cohen R, Cummings JL. Executive dysfunction and apathy predict functional impairment in Alzheimer disease. Am J Geriatr Psychiatry 2003;11: 214-221.

18. Starkstein SE, Petracca G, Chemerinski E, Kremer J. Syndromic validity of apathy in Alzheimer's disease. Am J Psychiatry 2001;158:872-877.

19. Tekin S, Fairbanks LA, O'Connor S, Rosenberg S, Cummings JL. Activities of daily living in Alzheimer's disease: neuropsychiatric, cognitive, and medical illness influences. Am J Geriatr Psychiatry 2001;9:81-86.

20. Rankin KP, Kramer JH, Mychack P, Miller BL. Double dissociation of social functioning in frontotemporal dementia. Neurology 2003;60:266-271.

21. Chow TW, Fridhandler JD, Binns MA, et al. Trajectories of behavioural disturbance in dementia. J Alzheimers Dis 2012;31:143-149.

22. Gregory CA. Frontal variant of frontotemporal dementia: a cross-sectional and longitudinal study of neuropsychiatric features. Psychol Med 1999;29:1205-1217.

23. Kertesz A, Blair M, McMonagle P, Munoz DG. The diagnosis and course of frontotemporal dementia. Alzheimer Dis Assoc Disord 2007;21:155-163.

24. Garre-Olmo J, Lopez-Pousa S, Vilalta-Franch J, de Gracia Blanco M, Vilarrasa AB. Grouping and trajectories of neuropsychiatric symptoms in patients with Alzheimer's disease: part II: two-year patient trajectories. J Alzheimers Dis 2010;22:1169-1180.

25. Marin DB, Green CR, Schmeidler J, et al. Noncognitive disturbances in Alzheimer's disease: frequency, longitudinal course, and relationship to cognitive symptoms. J Am Geriatr Soc 1997;45: 1331-1338.

26. Mesulam MM. Primary progressive aphasia: a language-based dementia. N Engl J Med 2003;349: $1535-1542$.

27. Snowden JS, Bathgate D, Varma A, Blackshaw A, Gibbons ZC, Neary D. Distinct behavioural profiles in frontotemporal dementia and semantic dementia. J Neurol Neurosurg Psychiatry 2001; 70:323-332.

28. Bathgate D, Snowden JS, Varma A, Blackshaw A, Neary D. Behaviour in frontotemporal dementia, Alzheimer's disease and vascular dementia. Acta Neurol Scand 2001;103:367-378.

29. Snowden JS, Neary D, Mann DMA. Frontotemporal dementia. Br J Psychiatry 2002;180:140-143.

30. Piguet O. Eating disturbance in behavioural-variant frontotemporal dementia. J Mol Neurosci 2011; 45:589-593.

31. Seeley WW, Bauer AM, Miller BL, et al. The natural history of temporal variant frontotemporal dementia. Neurology 2005;64:1384-1390.

32. Ikeda M, Brown J, Holland AJ, Fukuhara R, Hodges JR. Changes in appetite, food preference, and eating habits in frontotemporal dementia and Alzheimer's disease. J Neurol Neurosurg Psychiatry 2002;73:371-376.

33. Ahmed R, Irish M, Kam J, et al. Quantifying the eating abnormalities in frontotemporal dementia. JAMA Neurol 2014;71:1540-1546.

34. Hodges JR, Mitchell J, Dawson K, et al. Semantic dementia: demography, familial factors and survival in a consecutive series of 100 cases. Brain 2010;133:300-306.

35. Mioshi E, Hodges JR. Rate of change of functional abilities in frontotemporal dementia. Dement Geriatr Cogn Disord 2009;28:419-426.

36. Shinagawa S. Phenotypic variety in the presentation of frontotemporal lobar degeneration. Int Rev Psychiatry 2013;25:138-144.

37. Mioshi E, Foxe D, Leslie F, et al. The impact of dementia severity on caregiver burden in frontotemporal dementia and Alzheimer disease. Alzheimer Dis Assoc Disord 2013;27:68-73.

38. Hsieh S, Leyton C, Caga J, et al. The evolution of caregiver burden in frontotemporal dementia with and without amyotrophic lateral sclerosis. J Alzheimers Dis 2015;49:875-885.

39. Wagmiller RL. A fixed effects approach to assessing bias in proxy reports. Int J Public Opin 2009;21: 477-505. 
40. Borroni B, Grassi M, Premi E, et al. Neuroanatomical correlates of behavioural phenotypes in behavioural variant of frontotemporal dementia. Behav Brain Res 2012;235:124-129.

Received January 29, 2016. Accepted in final form April 14, 2016.

\section{AUTHOR CONTRIBUTIONS}

C.M. O'Connor contributed to the design and conceptualization of the study, data collection, analysis and interpretation of the data, and drafting and revision of the manuscript. L. Clemson contributed to the design and conceptualization of the study and revision of the manuscript. M. Hornberger contributed to the revision of the manuscript. C.E. Leyton contributed to the analysis and interpretation of the data and revision of the manuscript. J.R. Hodges contributed to study supervision and revision of the manuscript. O. Piguet contributed to study supervision and revision of the manuscript. E. Mioshi contributed to the design and conceptualization of the study, data collection, analysis and interpretation of the data, study supervision, and revision of the manuscript.

\section{STUDY FUNDING}

Supported in part by funding to ForeFront, a collaborative research group dedicated to the study of frontotemporal dementia and motor neuron disease, from the National Health and Medical Research Council (NHMRC) (APP1037746) and the Australian Research Council (ARC) Centre of Excellence in Cognition and its Disorders (CE11000102). Supported in part by the Alzheimer Association (NIRP12-258380).

\section{DISCLOSURES}

C.M. O'Connor received funding for travel from the Alzheimer's Association and receives support from Alzheimer's Australia Dementia Research Foundation, Mary Frances Stephens Scholarship (University of Sydney), and Leslie Rich Scholarship (University of Sydney). L. Clemson serves on the editorial advisory board of Journal of Ageing and Health and receives support from National Health and Medical Research Council (NHMRC) and a University of Sydney Bridging Grant. M. Hornberger serves as an Associate Editor for Journal of Alzheimer's Disease and Section Editor for Frontier in Aging Neuroscience. C. E. Leyton serves as an Associate Editor for Journal of Alzheimer's Disease and receives support from DVC Fellowship, University of Sydney. J.R. Hodges serves on the Advisory Board for Nature Reviews, Neurology ${ }^{\circledR}$, as an Associate Editor for Journal of Alzheimer's Disease, and on the editorial advisory boards of Aphasiology, Cognitive Neuropsychology, Nature Reviews, Neurology and Clinical Neuroscience, Acta Neuropsychologica, and ALS Journal; receives publishing royalties for Cognitive Assessment for Clinicians (Oxford University Press, 2007) and Frontotemporal Dementia Syndromes (Cambridge University Press, 2007); and has received research support from Australian Research Council Federation fellowship and NHMRC. O. Piguet serves on the editorial boards of Frontiers in Dementia Research, Frontiers in Emotion Science, Behavioural Neurology, and Journal of Alzheimer's Disease and receives research support from NHMRC, Australian Research Council, and Velux Stiftung, Switzerland. E. Mioshi serves as an Associate Editor for BMC Neurology and on the editorial board of Dementia and Geriatric Cognitive Disorders and receives research support from Alzheimer Society (UK), and Alzheimer Association (USA). Full disclosure form information provided by the authors is available with the full text of this article at Neurology.org/cp.

\section{Related articles from AAN physician and patient resources}

\section{Neurology ${ }^{\circledR} \quad$ Neurology.org}

Presymptomatic cognitive decline in familial frontotemporal dementia: A longitudinal study July 26, 2016;87:384-391.

\section{Neurology Now ${ }^{\circledR}$ - Neurologynow.com}

Sunna's Story: A powerful documentary charts the anguish-and hope-of families with children who have a rare neurologic disease August/September 2016;12:7. 


\title{
Neurology ${ }^{\circ}$ Clinical Practice
}

\author{
Longitudinal change in everyday function and behavioral symptoms in frontotemporal \\ dementia \\ Claire M. O'Connor, Lindy Clemson, Michael Hornberger, et al. \\ Neurol Clin Pract 2016;6;419-428 Published Online before print June 17, 2016 \\ DOI 10.1212/CPJ.0000000000000264
}

This information is current as of June 17, 2016

$\begin{array}{ll}\begin{array}{l}\text { Updated Information \& } \\ \text { Services }\end{array} & \begin{array}{l}\text { including high resolution figures, can be found at: } \\ \text { http://cp.neurology.org/content/6/5/419.full.html }\end{array} \\ \text { References } & \text { This article cites } 40 \text { articles, } 5 \text { of which you can access for free at: } \\ & \text { http://cp.neurology.org/content/6/5/419.full.html\#\#ref-list-1 } \\ & \text { This article has been cited by } 3 \text { HighWire-hosted articles: } \\ & \text { http://cp.neurology.org/content/6/5/419.full.html\#\#otherarticles } \\ \text { Citations } & \text { This article, along with others on similar topics, appears in the } \\ & \text { following collection(s): } \\ \text { Assessment of cognitive disorders/dementia } \\ \text { http://cp.neurology.org//cgi/collection/assessment_of_cognitive_disord } \\ \text { ers_dementia } \\ \text { Frontotemporal dementia } \\ \text { http://cp.neurology.org//cgi/collection/frontotemporal_dementia } \\ \text { Information about reproducing this article in parts (figures,tables) or in } \\ \text { its entirety can be found online at: } \\ \text { http://cp.neurology.org/misc/about.xhtml\#permissions } \\ \text { Permissions \& Licensing } \\ \text { Information about ordering reprints can be found online: } \\ \text { http://cp.neurology.org/misc/addir.xhtml\#reprintsus }\end{array}$

Neurol Clin Pract is an official journal of the American Academy of Neurology. Published continuously since 2011, it is now a bimonthly with 6 issues per year. Copyright $\odot 2016$ American Academy of Neurology. All rights reserved. Print ISSN: 2163-0402. Online ISSN: 2163-0933.

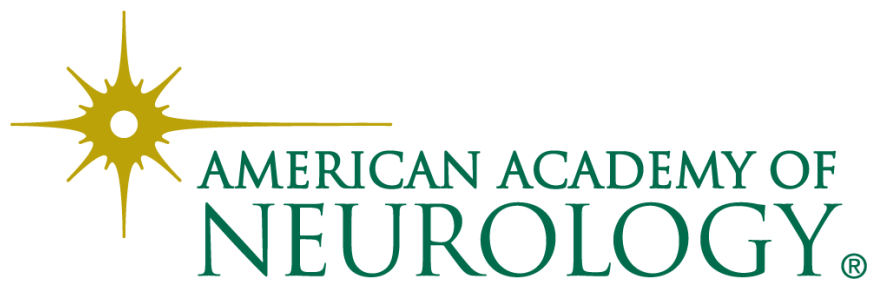

